\title{
S1.4
}

\section{IPOTESI PROGETTUALI PER IL RIASSETTO DELLA MICROBIOLOGIA CLINICA IN ITALIA}

\section{Magliano E.}

Presidente Associazione Microbiologi Clinici Italiani, Milano

L'assetto prospettico della Microbiologia Clinica in Italia, alla luce del riconoscimento della stretta interconnessione ed interazione tra diagnostica e clinica delle patologie da infezione, presuppone la necessità di pervenire, in tempi brevi, alla definizione di un modello organizzativo in rete, anche sulla base delle esperienze internazionali che sono descrittive di diversi approcci e strategie di centralizzazione e/o periferizzazione, comunque a distinti livelli di specializzazione, ma costantemente fondati su un postulato di base, costituito dall'autonomia della disciplina rispetto agli altri ambiti della Medicina di Laboratorio.

Come condizione preliminare alla realizzazione di un piano nazionale di settore, da proporre al Ministero della Salute e alla Istituzioni Regionali si è ritenuto indispensabile procedere ad un'indagine conoscitiva delle strutture di diagnostica microbiologica distribuite nelle varie aree del territorio nazionale.

A tale scopo l'AMCLI, d'intesa con la FESIM, ha realizzato una Indagine Conoscitiva "Strutture di diagnostica Microbiologica in Italia" con la collaborazione della Fondazione Smith-Kline, che si è conclusa nel dicembre 2004 con la pubblicazione di un report analitico descrittivo.

L'analisi è relativa a 295 schede di rilevazione idoneamente compilate pari al $23,4 \%$ dei questionari inviati a tutte le 1261 strutture sanitarie desunte dal database del Ministero della Salute.

L'indagine prevedeva di raccogliere, tra le altre, informazioni relative a:

1) Tipologie di strutture sanitarie presso cui è stata inserita la Diagnostica microbiologica;

2) Anagrafica della struttura;

3) Anno di istituzione;

4) Specializzazioni e posizione organizzativa del personale;

5) Tipologie e numero di esami eseguiti;

6) Descrizione di eventuali patologie e/o casistiche prevalenti;

7) Partecipazione a sistemi di sorveglianza;

8) Eventuale Status di Centro di riferimento;

9) Partecipazione a protocolli di Ricerca clinica;

10) Tipologia dei sistemi di garanzia della qualità.

I risultati consentono una prima valutazione sulla distribuzione delle strutture sanitarie nelle regioni e delle strutture di diagnostica microbiologica nelle strutture 
sanitarie.

Può essere altresì valutata l'entità e la distribuzione del personale dirigente dedicato in via esclusiva e non esclusiva e i livelli di specializzazione.

Un dato fondamentale è quello relativo alla incidenza delle prestazioni di microbiologia sul totale delle prestazioni di laboratorio, oltre ai livelli di complessità dei laboratori che le erogano.

Anche sulla base dei dati emersi dallo studio è possibile proporre un modello di organizzazione a rete di strutture microbiologiche ospedaliere e territoriali, a diverso livello di complessità, che operino in stretta integrazione con le strutture dedicate alla prevenzione, trattamento, cura e riabilitazione delle patologie da infezione, proponendosi sia obiettivi di maggiore appropriatezza ed efficacia degli interventi assistenziali che di miglioramento del rapporto costo-beneficio.

Tale rete andrà riconosciuta nei piani sanitari delle regioni

Tale architettura presuppone un ruolo esclusivo per lo specialista in microbiologia e virologia ed una organizzazione dei servizi indipendente dalle strutture afferenti alle altre discipline, dove le strutture di microbiologia siano interconnesse tra loro ai diversi livelli, secondo modelli di tipo dipartimentale. 